\title{
Is the predictive model for asthma development useful as a tool for diagnosing pediatric asthma?
}

Bong-Seong Kim, MD, PhD

Department of Pediatrics, Gangneung Asan Hospital, University of Ulsan College of Medicine, Gangneung, Korea

Asthma is known to be underdiagnosed in 18\%-75\% of children with asthma. Likewise, the overdiagnosis of asthma is also a common issue. ${ }^{1)}$ In $80 \%$ of cases, children develop asthma before the age of 6 years, usually before the age of 3 years. ${ }^{2)}$ Young child. ren who are likely to develop asthma can benefit from both timely asthma diagnosis and preventive interventions even if their asthma is resolved later in life. ${ }^{3)}$ However, the diagnosis of asthma in preschool children has not yet been standardized, and it is seemingly difficult to perform some current tests when diagnosing asthma. ${ }^{4)}$ Most clinicians practically diagnose asthma at preschool age based on subjective clinical symptoms.

Asthma is nowadays understood as a heterogeneous disease, a complex syndrome, or an umbrella term that is used to refer to patients with similar spectra of symptoms but with diverse or uncertain etiopathogenesis. ${ }^{5)}$ In this context, a diagnosis of asthma should depend on the consensus achieved in the scientific community. Accordingly, such a consensus is usually embodied in the clinical practice guidelines, most of which (including the Global Initiative for Asthma) are determined by a group of experts selected on a structured basis. ${ }^{4}$ The guidelines are usually linked to the diagnosis of asthma in preschool children based on the presence of compatible symptoms (several episodes of bronchial obstruction, preferably witnessed by a doctor), appropriate response to treatment and exclusion of other alternative diagnoses. $^{4)}$

Several predictive models have been developed to predict whether preschool children will have asthma at school age, such as the stringent and loose forms of the Asthma Predictive Index (API). ${ }^{6,7)}$ The API was developed 20 years ago using data from the Tucson Children's Respiratory Study birth cohort and is currently applied partially in Korea and is used to predict asthma. 7) It is necessary to examine whether the API is useful or not when applied in Korea, since the API has been determined and evaluated by the standards of epidemiological studies in other countries.

Therefore, the study conducted by Lee et al. $\left.{ }^{8}\right)$ published in the current issue reveals the association between current asthma, the API and other tests. It also verifies the diagnostic accuracy of the
API.

This study showed that the questionnaire-based diagnosis of current asthma is fairly associated with the API, but not with spirometry, airway hyperreactivity (AHR), fractional expiratory nitric oxide, or atopic sensitization in preschool children. ${ }^{8)}$ This study seems to be limited by its cross-sectional design and the small number of included current asthma patients. In addition, AHR or atopic sensitization tests were performed in a small number of asthma patients, making it difficult to draw conclusions. However, as the authors suggested, asthma in preschool children may differ from that in late childhood or adult patient.

In this study, the loose and stringent APIs had high negative predictive values (NPVs) of $98.7 \%$ and $98.6 \%$, respectively but conversely had low positive predictive values (PPVs) of $12.7 \%$ and $14.1 \%{ }^{8)}$ The NPVs reported in this study were similar to those reported in the original study that showed the usefulness of the API; however, the PPVs in this study were lower. ${ }^{9}{ }^{9}$ In contrast, the API showed higher sensitivity and specificity in this study than in the original study; therefore, it can be considered applicable to patients with recurrent wheezing. ${ }^{9}$ )

Although predictive models for the development of childhood asthma have been designed to facilitate the diagnosis and prevention of asthma, their usefulness for pediatric asthma remains unclear in clinical practice. ${ }^{10)}$ To be clinically valuable, such predictive models must have high PPV and high sensitivity. $\left.{ }^{6}\right)$ High PPV indicates that a child with high predictive risk will actually develop asthma. High sensitivity allows the model to identify most children who will develop asthma in the future. ${ }^{6,10)}$ All predictive models developed to date have low PPVs or low sensitivity. Currently, such models do not have sufficient accuracy for routine clinical use. ${ }^{10)}$ Nevertheless, the API is a simpler and less expensive tool for identifying children at risk of developing asthma. It is easy to apply, has been validated in different populations, and shown to improve patient outcomes used by busy clinicians. ${ }^{7)}$

In conclusion, predictive models for the development of childhood asthma, such as the API, are considerably helpful in the diagnosis and prevention of asthma but may not be enough to

Corresponding author: Bong-Seong Kim, MD, PhD. Department of Pediatrics, Gangneung Asan Hospital, University of Ulsan College of Medicine, 38, Bangdong-gil, Sacheon-myeon, Gangneung 25440, Korea 
replace the diagnostic process of childhood asthma. At present, predictive models for the development of asthma are valuable as a supportive role to clinical diagnosis based on the guidelines. Through further research, predictive models of asthma need to be improved and it is necessary to conduct a prospective study by applying the API or other predictive models for asthma in Korean preschoolers to confirm its effectiveness.

\section{Conflicts of interest}

No potential conflict of interest relevant to this article was reported.

See the article "Asthma predictive index as a useful diagnostic tool in preschool children: a cross-sectional study in Korea" in Volume 63 on page 104.

\section{References}

1. Nolte H, Nepper-Christensen S, Backer V. Unawareness and undertreatment of asthma and allergic rhinitis in a general population. Respir Med 2006;100:354-62.
2. Martinez FD. Development of wheezing disorders and asthma in preschool children. Pediatrics 2002;109(2 Suppl):362-7.

3. Andersson M, Hedman L, Bjerg A, Forsberg B, Lundbäck B, Rönmark E. Remission and persistence of asthma followed from 7 to 19 years of age. Pediatrics 2013;132:e435-42.

4. Moral L, Vizmanos G, Torres-Borrego J, Praena-Crespo M, TortajadaGirbés M, Pellegrini FJ, et al. Asthma diagnosis in infants and preschool children: a systematic review of clinical guidelines. Allergol Immunopathol (Madr) 2019;47:107-21.

5. Gauthier M, Ray A, Wenzel SE. Evolving concepts of asthma. Am J Respir Crit Care Med 2015;192:660-8.

6. Savenije OE, Kerkhof M, Koppelman GH, Postma DS. Predicting who will have asthma at school age among preschool children. J Allergy Clin Immunol 2012;130:325-31.

7. Castro-Rodriguez JA. The Asthma Predictive Index: a very useful tool for predicting asthma in young children. J Allergy Clin Immunol 2010;126: 212-6.

8. Lee DH, Kwon JW, Kim HY, Seo JH, Kim HB, Lee SY, et al. Asthma Predictive index as a useful diagnostic tool in preschool children: a crosssectional study in Korea. Clin Exp Pediatr 2020;63:104-9.

9. Castro-Rodríguez JA, Holberg CJ, Wright AL, Martinez FD. A clinical index to define risk of asthma in young children with recurrent wheezing. Am J Respir Crit Care Med 2000;162(4 Pt 1):1403-6.

10. Luo G, Nkoy FL, Stone BL, Schmick D, Johnson MD. A systematic review of predictive models for asthma development in children. BMC Med Inform Decis Mak 2015;15:99. 